\title{
Limits for the Use of Auto-Balancing
}

\author{
Karl-Olof Olsson \\ Department of Mechanical Engineering, Linköping University, Linköping, Sweden
}

The principle for autobalancing by the use of balls running freely in a ring concentric with the rotor center has been known for a long time. However, only in recent times has it been used in real machinery, such as grinding machines and laundry machines. By the increased need for higher precision, it is likely to come into use more frequently in the future. This means that it is important to know its application possibilities better. By using a time averaging technique, it has been possible to find analytical solutions to a number of problems. This article deals with; (1) the case of a rotor with anisotropic bearings and damping, where precise conditions for possible balancing are presented; (2) influence from inaccuracy of balancing ring and a rotor with longitudinal extension and thus having not only a point mass but also a moment of inertia; and (3) using two balancing planes to allow both static and dynamic balancing.

Keywords Automatic balancing, Rotor dynamics, Stability, Vibrations

Rotating machinery is one of the most frequent reasons for vibrations. There are many mechanisms behind the vibrations, but the most common is by far that of unbalance. In order to limit the distubance from this many rotors, and in particular those rotating at high speed, rotors are being balanced, mostly as part of their manufacture. Field balancing is often also necessary in order to finally adjust the state of the rotor or for compensating changes that might have occurred. Active balancing might be a possible method, see e.g., Gosiewski (1985a, 1985b), and Ohtami et al. (2001). Another alternative is a mechanism that automatically adjusts the balancing state, referred to as autobalancing. There are primarily two applications for this alternative: (1) when extreme accuracy is necessary (disc drives may be an

Received 30 March 2003; accepted 30 June 2003.

Address correspondence to Karl-Olof Olsson, Department of Mechanical Engineering, Linköping University, SE-581 83 Linköping, Sweden. E-mail: karlolof.olsson@telia.com example), and (2) when the state of balance is continously or intermittently changed. A typical example of this is the centrifuge for a laundry machine, however fans, grinding wheels, and separators also belong to this category.

The most well-known device for autobalancing is when two (or more) balls are running freely in a ring concentric with the rotor. This was first described by Thearle (1932), and referred to by others, e.g., Den Hartog (1954) and Lindell (1996) who describes how it is used to reduce the discomfort caused by grinding machines. Two or more pendulums can also be used, see Kubo et al. (1986). It is known that these devices can work only for rotors running supercritically.

This article deals with the method of free balls. The basic theory includes any number of balls, but is valid only if the balls stay out of contact with each other. All implementations of the theory, however, concentrate on the simplest case with only two balls. By assuming that the motions of the balls are slow as compared to the rotational speed, time averaging can be performed which greatly simplfies the analysis. It is known, see e.g., Inoue (1978), Rajalingham (1998), and Sharp (1975), that there are regions where the balls will not find their equilibrium positions but will continue to rotate. This has also been confirmed through direct simulation, see Wettergren (2001). This phenomenon cannot be dealt with in a stringent way using the theory put forward here.

\section{BASIC THEORY}

\section{Geometry}

In Figure 1, the basic arrangement is demonstrated but showing only one ball, having mass $m_{b}$. The motion of the rotor is described in a stationary $x, y, z$-system. The initial unbalance, $\theta_{0}$, is directed along the rotating $\xi$-axis. The balls change the unbalance and place the center of gravity defined by $\theta_{\xi}$ and $e_{\eta}$. The position of the ball is given by the angle $\varphi$. It runs in a circle with radius $r_{0}$, but a small deviation is also added from the ideal geometry given by

$$
\begin{aligned}
r & =r_{0}+\Delta r \\
\Delta r & =\sum \Delta r_{n} \cdot \cos n\left(\varphi-\alpha_{n}\right)
\end{aligned}
$$




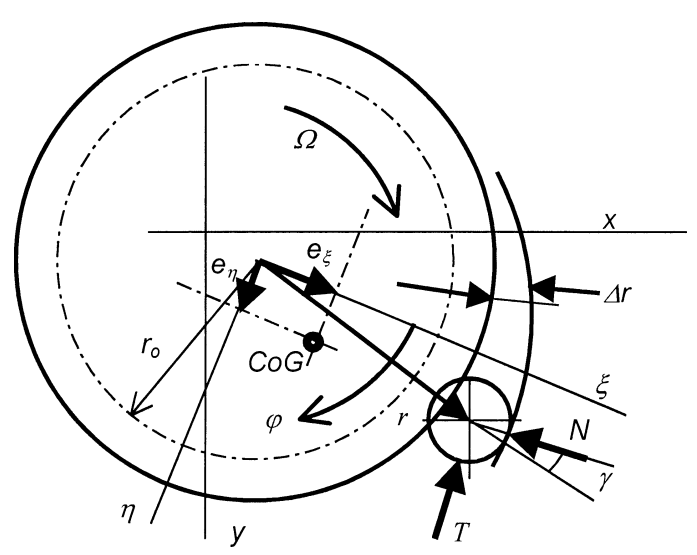

FIGURE 1

Basic geometry.

which also defines the angle, $\gamma$, to the normal force, $N$, and the force, $T$, acting as a resistance to the motion of the ball.

$$
\gamma=\frac{1}{r_{0}} \sum-\Delta r_{n} \cdot n \cdot \sin n\left(\varphi-\alpha_{n}\right)
$$

It is easily recognized that the eccentricities in $\xi$ - and $\eta$ directions become

$$
\begin{aligned}
& e_{\xi}=e_{0} \cdot\left(1+\kappa \sum \cos \varphi_{i}\right) \\
& e_{\eta}=e_{0} \cdot \kappa \sum \sin \varphi_{i}
\end{aligned}
$$

where

$$
\kappa=\frac{r_{0} \cdot m_{b}}{e_{0} \cdot m}
$$

and $m$ is the total mass of the rotor including the balls. The eccentricities can also be described as

$$
\begin{aligned}
& e_{\xi}=e \cdot \cos \beta \\
& e_{\eta}=e \cdot \sin \beta
\end{aligned}
$$

with

$$
\begin{aligned}
e & =\sqrt{e_{\xi}^{2}+e_{\eta}^{2}} \\
\tan \beta & =\frac{e_{\eta}}{e_{\xi}}
\end{aligned}
$$

\section{Dynamics of Rotor}

Along with the assumption that the motions of the balls are slow, it is justified to use the steady-state solution for the motion of the rotor. It can now be described by

$$
\begin{aligned}
& x=x_{0} \cdot \cos \left(\Omega t+\beta-\psi_{x}\right) \\
& y=y_{0} \cdot \sin \left(\Omega t+\beta-\psi_{y}\right)
\end{aligned}
$$

where

$$
\begin{aligned}
& x_{0}=e \cdot \Phi_{x} \\
& y_{0}=e \cdot \Phi_{y}
\end{aligned}
$$

Here, $\Phi_{x}$ and $\Phi_{y}$ are the magnification factors and $\psi_{x}$ and $\psi_{y}$ are the corresponding phase angles. These quantities can be found in any textbook and are thus omitted here due to limited space. Using Equations (3), (5) and (6) we arrive at

$$
\begin{aligned}
& x=\Phi_{x}\left[e_{\xi} \cdot \cos \left(\Omega t-\psi_{x}\right)-e_{\eta} \cdot \sin \left(\Omega t-\psi_{x}\right)\right] \\
& y=\Phi_{y}\left[e_{\xi} \cdot \sin \left(\Omega t-\psi_{y}\right)+e_{\eta} \cdot \cos \left(\Omega t-\psi_{y}\right)\right]
\end{aligned}
$$

\section{Dynamics of Ball}

The positions of the balls are obviously

$$
\begin{aligned}
& x_{b}=x+r \cdot \cos (\Omega t+\varphi) \\
& y_{b}=y+r \cdot \sin (\Omega t+\varphi)
\end{aligned}
$$

The motion of the ball is governed by

$$
\begin{aligned}
& -N \cdot \cos (\Omega t+\varphi-\gamma)+T \cdot \sin (\Omega t+\varphi-\gamma)=m_{b} \cdot \ddot{x}_{b} \\
& -N \cdot \sin (\Omega t+\varphi-\gamma)-T \cdot \cos (\Omega t+\varphi-\gamma)=m_{b} \cdot \ddot{y}_{b}
\end{aligned}
$$

Eliminating $N$ between these equations gives

$$
T=m_{b} \cdot \ddot{x}_{b} \sin (\Omega t+\varphi-\gamma)-m_{b} \cdot \ddot{y}_{b} \cos (\Omega t+\varphi-\gamma)
$$

By using Equation (7) we finally arrive at

$$
\begin{aligned}
T= & m_{b} \cdot\left\{\Phi_{x} \cdot \Omega^{2}\left[-e_{\xi} \cdot \cos \left(\Omega t-\psi_{x}\right)+e_{\eta} \cdot \sin \left(\Omega t-\psi_{x}\right)\right]\right. \\
& \left.+r\left\lfloor-\cos (\Omega t+\varphi) \cdot(\Omega t+\dot{\varphi})^{2}-\sin (\Omega t+\varphi) \cdot \ddot{\varphi}\right]\right\} \\
& \times\{\sin (\Omega t+\varphi)-\gamma \cdot \cos (\Omega t+\varphi)\}-m_{b} \\
& \times\left\{\Phi_{y} \cdot \Omega^{2}\left[-e_{\eta} \cdot \cos \left(\Omega t-\psi_{y}\right)-e_{\xi} \cdot \sin \left(\Omega t-\psi_{y}\right)\right]\right. \\
& \left.+r\left[-\sin (\Omega t+\varphi) \cdot(\Omega+\dot{\varphi})^{2}+\cos (\Omega t+\varphi) \cdot \ddot{\varphi}\right]\right\} \\
& \times\{\cos (\Omega t+\varphi)+\gamma \cdot \sin (\Omega t+\varphi)\}
\end{aligned}
$$

We now utilize the assumption that $\varphi$ is changing slowly, i.e., $\dot{\varphi} \ll \Omega$. This implies that we can allow a time-averaging over one period of revolution. In doing so, and also recognizing that $\gamma \ll 1$ and $\Delta r \ll r_{0}$, we obtain

$$
\begin{aligned}
r_{0} \ddot{\varphi}+ & \frac{T}{m_{b}}-r_{0} \cdot \Omega^{2} \cdot \gamma \\
= & \frac{\Omega^{2}}{2}\left[-\sin \varphi \cdot\left(\Phi_{x} \cdot \cos \psi_{x} \cdot e_{\xi}+\Phi_{x} \cdot \sin \psi_{x} \cdot e_{\eta}\right.\right. \\
& \left.\left.+\Phi_{y} \cdot \sin \psi_{y} \cdot e_{\eta}+\Phi_{y} \cdot \cos \psi_{y} \cdot e_{\xi}\right)\right] \\
& +\frac{\Omega^{2}}{2}\left[\operatorname { c o s } \varphi \cdot \left(-\Phi_{x} \cdot \sin \psi_{x} \cdot e_{\xi}+\Phi_{x} \cdot \cos \psi_{x} \cdot e_{\eta}\right.\right. \\
& \left.\left.+\Phi_{y} \cdot \cos \psi_{y} \cdot e_{\eta}-\Phi_{y} \cdot \sin \psi_{y} \cdot e_{\xi}\right)\right]
\end{aligned}
$$


This equation is of course valid for every single ball. If we rearrange it and also utilize Equation (2), we finally arrive at

$$
\begin{aligned}
& r_{0} \ddot{\varphi}_{i}+\frac{T_{i}}{m_{b}}-r_{0} \cdot \Omega^{2} \cdot \gamma=e_{0} \cdot \frac{\Omega^{2}}{2} \cdot \Phi_{c}\left[-\sin \varphi_{i}\right. \\
& \left.+\kappa\left(-\sin \varphi_{i} \cdot \sum_{n} \cos \varphi_{j}+\cos \varphi_{i} \cdot \sum_{n} \sin \varphi_{j}\right)\right] \\
& +e_{0} \cdot \frac{\Omega^{2}}{2} \cdot \Phi_{s}\left[-\cos \varphi_{i}-\kappa\left(\sin \varphi_{i} \cdot \sum_{n} \sin \varphi_{j}\right.\right. \\
& \left.\left.\quad+\cos \varphi_{i} \cdot \sum_{n} \cos \varphi_{j}\right)\right]
\end{aligned}
$$

where

$$
\begin{aligned}
& \Phi_{c}=\left(\Phi_{x} \cdot \cos \psi_{x}+\Phi_{y} \cdot \cos \psi_{y}\right) \\
& \Phi_{s}=\left(\Phi_{x} \cdot \sin \psi_{x}+\Phi_{y} \cdot \sin \psi_{y}\right)
\end{aligned}
$$

\section{APPLICATIONS}

In this article we will restrict ourselves to cases with only two balls. We also assume that $T$ has a viscous character, i.e., $T=c \cdot r_{0} \cdot \dot{\varphi}$. Now Equation (8) reduce to

$$
\begin{aligned}
r_{0} \ddot{\varphi}_{1} & +\frac{c \cdot r_{0}}{m_{b}} \dot{\varphi}-r_{0} \Omega^{2} \gamma=e_{0} \frac{\Omega^{2}}{2}\left\{\Phi _ { c } \left[-\sin \varphi_{1}+\kappa \sin \left(\varphi_{2}\right.\right.\right. \\
& \left.\left.\left.-\varphi_{1}\right)\right]+\Phi_{s}\left[-\cos \varphi_{1}-\kappa\left(1+\cos \left(\varphi_{2}-\varphi_{1}\right)\right)\right]\right\}
\end{aligned}
$$

for ball number 1 and, correspondingly, for the other ball.

\section{Perfect Balancing Ring}

The ring without geometrical defects is defined by $\Delta r=$ $\gamma=0$.

\section{Equilibrium}

For the simple case of no damping $\left(\Phi_{s}=0\right)$ there are two possible equilibrium solutions to Equation (10). The first, $\mathbf{A}$, is

$$
\varphi_{1}=\varphi_{2}=0
$$

and the second, $\mathbf{B}$, is

$$
\varphi_{1}=-\varphi_{2} \quad \text { and } \quad \cos \varphi_{1}=\cos \varphi_{2}=-\frac{1}{2 \kappa}
$$

In order for this to be meaningful we must have $\kappa>0.5$ which simply means that the balls must be large enough to be able to counteract the unbalance. Solution A means that the balls are positioned in the direction of the initial unbalance and thus aggravating it. For the case including damping, the solution is a bit more complicated but it is still characterized by $\varphi_{1}=\varphi_{2}$ and the balls positioned to aggravate the unbalance. The details have been left aside as they are not of too much interest. Solution $\mathbf{B}$ is generally true and is the well-known condition for effective balancing. Which of these two solutions will materialize is of course depending on their stability.

\section{Stability}

If we give the ball a small change of position, $\Delta \varphi$, from its equilibrium, we obtain from Equation (10)

$$
\begin{aligned}
& r_{0} \ddot{\varphi}_{1}+\frac{c \cdot r_{0}}{m_{b}} \cdot \dot{\varphi}=e_{0} \cdot \frac{\Omega^{2}}{2}\left\{\Phi_{c}\left[-\cos \varphi_{1}-\kappa \cos \left(\varphi_{2}-\varphi_{1}\right)\right]\right. \\
& \left.+\Phi_{s}\left[\sin \varphi_{1}+\kappa \sin \left(\varphi_{2}-\varphi_{1}\right)\right]\right\} \cdot \Delta \varphi
\end{aligned}
$$

For the two equilibrium solutions this reduces to

$$
\begin{aligned}
& r_{0} \ddot{\varphi}_{1}+\frac{c \cdot r_{0}}{m_{b}} \cdot \dot{\varphi}=-e_{0} \cdot \frac{\Omega^{2}}{2} \cdot \Phi_{c} \cdot(1+\kappa) \cdot \Delta \varphi \\
& r_{0} \ddot{\varphi}_{1}+\frac{c \cdot r_{0}}{m_{b}} \cdot \dot{\varphi}=e_{0} \cdot \frac{\Omega^{2}}{2} \cdot \Phi_{c} \cdot \kappa \cdot \Delta \varphi
\end{aligned}
$$

for case $\mathbf{A}$ (no damping provided even here) and $\mathbf{B}$ (generally valid), respectively.

The factor before $\Delta \varphi$ must be $<0$ in order for the solution to be stable. This means that $\mathbf{A}$ is stable when $\Phi_{c}>0$, which means subcritical operation, while $\mathbf{B}$ is stable when $\Phi_{c}<0$, meaning supercritical operation. This confirms well-known facts. For an anisotropic case there are two critical speeds, $\omega_{e x}$ and $\omega_{e y}$, assuming $\omega_{e x}<\omega_{e y}$. The interpretation now is that $\mathbf{A}$ is stable below $\omega_{e x}$ and $\mathbf{B}$ is stable above $\omega_{e y}$. In between these two, $\mathbf{B}$ starts to be stable just above $\omega_{e x}$. At some point $\Phi_{c}$ changes sign. This is the point when the $x$ - and $y$-amplitudes have the same magnitude. Above that, solution $\mathbf{A}$ is again the stable one. This finding confirms analytically the results obtained through simulation by Wettergren (2001).

\section{Transients}

To follow the balls from some initial position to the stable equilibrium, Equation (10) must be solved numerically. This will be done for the simplest case where $\Phi_{x}=\Phi_{y}=\Phi$ and $\psi_{x}=\psi_{y}=0$. The equations are favorably transformed to nondimensional form by introducing the nondimensional time $\tau=\Omega t$. In doing so, we come to

$$
\varphi_{1}^{\prime \prime}+\lambda \cdot \varphi_{1}^{\prime}=u\left[-\sin \varphi_{1}+\kappa \cdot \sin \left(\varphi_{2}-\varphi_{1}\right)\right]
$$

where' signifies differentiation with respect to $\tau, \lambda=c / m_{b} / \Omega$ is a type of nondimensional damping for the ball, and $u=e_{0} / r_{0}$. $\Phi_{c}$ is a quantity defining the strength of the vibration caused by the initial unbalance. Rough estimation indicates that $\lambda$ is typically of the order $0.1-1$, whilst $|u|$ may vary from 0.00001 for a precision machinery running well above critical up to 10 for 


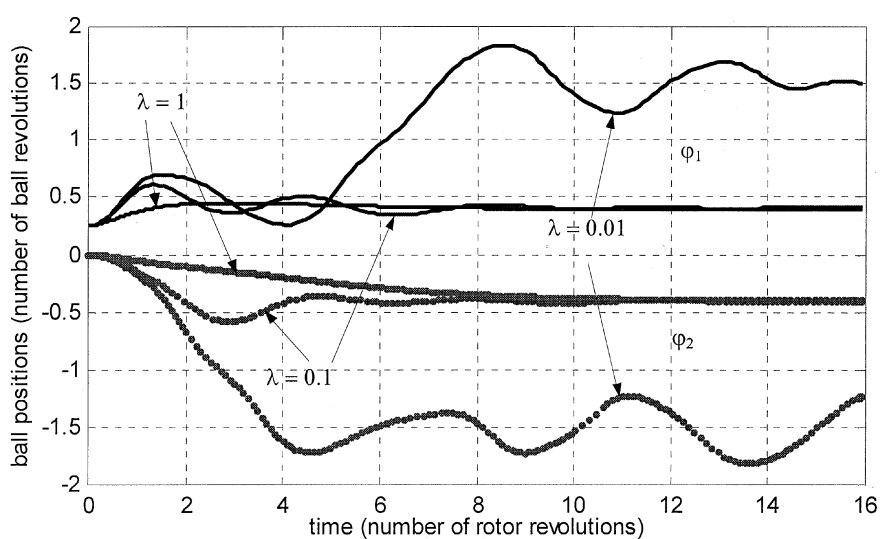

FIGURE 2

Ball transients shown as ball motions vs. rotor revolutions.

$$
u=-0.1, \kappa=0.6 \text {. }
$$

a badly balanced machine (e.g., a laundry centrifuge) running close to its critical.

Figure 2 shows the motion of the balls for a case with large unbalance, $\mathrm{u}=-0.1$. For high damping, $\lambda=1$, the balls move directly to their final positions, which is $\varphi_{1}=-\varphi_{2}=146.4^{\circ}$. For lower damping they make a few oscillations before equilibrium is reached. For even lower damping the balls perform a more violent motion and even after 16 rotor revolutions they have not come to rest. It should be noted that the motion as shown would mean that they would collide with each other. (This could in principle be avoided by letting the two balls have separate tracks.) It is also doubtful if the assumption of slow ball motion is justified for $\lambda=0.01$. When the unbalance has a smaller value, the motion is much slower and often requires many turns of the rotor before equilibrium is reached.

\section{Balancing Ring with Geometrical Defects}

When the balancing ring has deviations from ideal geometry it will not be possible to reach a perfect balancing. It is difficult to give a generally applicable answer to what a defect might imply, therefore two typical types of errors will be analyzed: eccentricity and ellipticity. We restrict ourselves again to the simple case where $\Phi_{x}=\Phi_{y}=\Phi$ and $\psi_{x}=\psi_{y}=0$.

\section{Eccentric Ring}

The eccentric ring is described by $\Delta r_{1} \neq 0$ and all other $\Delta r_{v}=0$. The equation to be solved is now

$$
\begin{aligned}
& r_{0} \ddot{\varphi}_{1}+\frac{c \cdot r_{0}}{m_{b}} \cdot \dot{\varphi}+\Omega^{2} \cdot \Delta r_{1} \cdot \sin \left(\varphi_{1}-\alpha\right) \\
& \quad=e_{0} \cdot \Omega^{2} \cdot \Phi\left[-\sin \varphi_{1}+\kappa \cdot \sin \left(\varphi_{2}-\varphi_{1}\right)\right]
\end{aligned}
$$

and, correspondingly, for the other ball.

It is advantageous to introduce $\varphi_{m}=\left(\varphi_{1}+\varphi_{2}\right) / 2$ and $\Delta \varphi=$ $\left(\varphi_{1}-\varphi_{2}\right) / 2$. With these notations the resulting unbalances can be written

$$
\begin{aligned}
& e_{\xi}=e_{0} \cdot\left[1+2 \kappa \cdot \cos \varphi_{m} \cdot \cos \Delta \varphi\right] \\
& e_{\eta}=e_{0} \cdot 2 \kappa \cdot \sin \varphi_{m} \cdot \cos \Delta \varphi
\end{aligned}
$$

After simple but tedious manipulations, and using $r_{1 c}=\Delta r_{1}$. $\cos \alpha$ and $r_{1 s}=\Delta r_{1} \cdot \sin \alpha$, we arrive at the solution

$$
\begin{aligned}
\tan \varphi_{m} & =\frac{r_{1 s}}{e_{0} \cdot \Phi+r_{1 c}} \\
\cos \Delta \varphi & =-\frac{1}{2 \kappa \cdot e_{0} \cdot \Phi^{2}} \cdot \sqrt{\left(e_{0} \cdot \Phi+r_{1 c}\right)^{2}+r_{1 s}{ }^{2}}
\end{aligned}
$$

The final unbalance can then be deduced to

$$
e_{\xi}=-r_{1 c} / \Phi \text { and } e_{\eta}=-r_{1 s} / \Phi
$$

For the rotor this means that it will vibrate with an amplitude exactly the same as the eccentricity of the ring, but in the opposite direction. For the ring it means that it rotates with its center stationary. This result can also be arrived at by a simpler reasoning. It reflects the simple fact that this is the only possibility for the balls to be at rest.

\section{Ellipticity}

The elliptic ring is described by $\Delta r_{2} \neq 0$ and all other $\Delta r_{v}=$ 0 . The equation to be solved is now

$$
\begin{gathered}
r_{0} \ddot{\varphi}_{1}+\frac{c \cdot r_{0}}{m_{b}} \cdot \dot{\varphi}+2 \Omega^{2} \Delta r_{2} \cdot \sin 2\left(\varphi_{1}-\alpha\right) \\
=e_{0} \Omega^{2} \Phi\left[-\sin \varphi_{1}+\kappa \cdot \sin \left(\varphi_{2}-\varphi_{1}\right)\right]
\end{gathered}
$$

and, correspondingly, for the other ball.

This can only be solved numerically. To demonstrate the solutions, only the two fundamental cases with $\alpha=0$ (ellipticity directed towards the initial unbalance) and $\alpha=\pi / 2$ (ellipticity orthogonal to the initial unbalance). Stable equilibrium positions and their resulting unbalances are shown in Table 1. There are also in some of the cases a number of other equilibrium positions which are not stable. They are not shown here.

\section{Rotor with Axial Extension}

Thus far the rotor has been regarded as a point mass. All real rotors also have an extension and, consequently, a moment of inertia. This also means that they have two or more natural frequencies and dynamic as well as static unbalance. In order to balance such rotors one must have two balancing planes and in order for the auto-balancing to work there must be two balancing rings, here located at axial positions $z_{1}$ and $z_{2}$. Figure 3 shows such a rotor. The rotor body is assumed to be rigid. We assume an isotropic and undamped case for simplicity. Its motion can be described by movements in the $x$-direction together with a tilting, $\theta$.

For the most simple case with no coupling between $x$ and $\theta$, the motion can be decribed by

$$
\begin{aligned}
& x=\Phi_{x}\left(e_{\xi} \cos \Omega t-e_{\eta} \sin \Omega t\right) \\
& \theta=\Phi_{\theta}\left(\vartheta_{\xi} \cos \Omega t-\vartheta_{\eta} \sin \Omega t\right)
\end{aligned}
$$


TABLE 1

Results of Balancing with an Elliptic Ring. Stable Ball

Positions and Residual Unbalances. $\kappa=0.6$

\begin{tabular}{|c|c|c|c|c|c|c|c|}
\hline \multicolumn{4}{|c|}{$\alpha=0$} & \multicolumn{4}{|c|}{$\alpha=90$} \\
\hline$\Delta r_{1} / e_{0}$ & $\varphi_{1}$ & $\varphi_{2}$ & $e / e_{0}$ & $\Delta r_{1} / e_{0}$ & $\varphi_{1}$ & $\varphi_{2}$ & $e / e_{0}$ \\
\hline \multirow[t]{3}{*}{1} & 0 & 0 & 2.2 & 1.0 & 101.1 & -101.1 & 0.769 \\
\hline & 0 & 180 & 1.0 & & 104.5 & 104.5 & 1.356 \\
\hline & 180 & 180 & 0.2 & & -104.5 & -104.5 & 1.356 \\
\hline \multirow[t]{2}{*}{0.5} & 0 & 180 & 1.0 & 0.5 & 108.2 & -108.2 & 0.623 \\
\hline & 180 & 180 & 0.2 & & $\begin{array}{r}120 \\
-120\end{array}$ & $\begin{array}{r}120 \\
-120\end{array}$ & $\begin{array}{l}1.114 \\
1.114\end{array}$ \\
\hline 0.1 & 180 & 180 & 0.2 & 0.1 & 128.7 & -128.7 & 0.249 \\
\hline 0.01 & 149.5 & -149.5 & 0.034 & 0.01 & 143.8 & -143.8 & 0.031 \\
\hline 0.001 & 146.7 & -146.7 & 0.003 & 0.001 & 146.2 & -146.2 & 0.003 \\
\hline
\end{tabular}

where $\Phi_{x}$ and $\Phi_{\theta}$ are the magnification factors and $\vartheta_{\xi}$ and $\vartheta_{\eta}$ are the dynamic unbalances. The unbalances consist of initial values $e_{0}$, and $\vartheta_{0}$, respectively, to which the influences from the balancing balls are added.

They are

$$
\begin{aligned}
& e_{\xi}=e_{0}\left[1+\kappa_{1} \sum c_{1}+\kappa_{2} \sum c_{2}\right] \\
& e_{\eta}=e_{0}\left[\kappa_{1} \sum s_{1}+\kappa_{2} \sum s_{2}\right] \\
& \vartheta_{\xi}=\vartheta_{0}\left[1+\Gamma_{1} \sum c_{1}+\Gamma_{2} \sum c_{2}\right] \\
& \vartheta_{\eta}=\vartheta_{0}\left[\Gamma_{1} \sum s_{1}+\Gamma_{2} \sum s_{2}\right]
\end{aligned}
$$

with

$$
\begin{aligned}
\sum c_{1} & =\left(\cos \varphi_{11}+\cos \varphi_{12}\right) \\
\sum c_{2} & =\left(\cos \varphi_{21}+\cos \varphi_{22}\right) \\
\sum s_{1} & =\left(\sin \varphi_{11}+\sin \varphi_{12}\right) \\
\sum s_{2} & =\left(\sin \varphi_{21}+\sin \varphi_{22}\right)
\end{aligned}
$$

Here, $\varphi_{11}, \varphi_{12}$ and $\varphi_{21}, \varphi_{22}$ are the positions of the balls in the two balancing rings, $\kappa_{1}$ and $\kappa_{2}$ have meaning corresponding to the basic theory previously discussed, and

$$
\Gamma_{1}=\frac{m e_{0} z_{1} \kappa_{1}}{j \vartheta_{0}} \quad \text { and } \quad \Gamma_{2}=\frac{m e_{0} z_{2} \kappa_{2}}{J \vartheta_{0}}
$$

with $J$ being the moment of inertia.

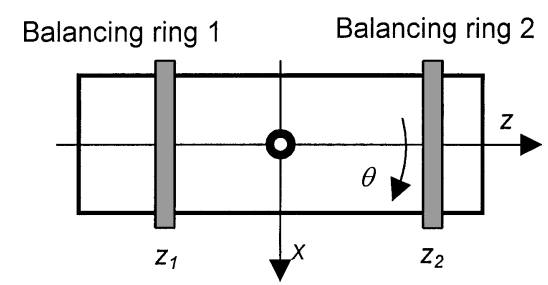

FIGURE 3

Rotor with axial extension.
The motion at balancing ring 1 is obviously

$$
\begin{aligned}
x_{1}= & x+\theta \cdot z_{1}=\Phi_{x}\left\lfloor e_{\xi} \cos \Omega t-e_{\eta} \sin \Omega t\right\rfloor \\
& +z_{1} \Phi_{\theta}\left\lfloor\vartheta_{\xi} \cos \Omega t-\vartheta_{\eta} \sin \Omega t\right\rfloor
\end{aligned}
$$

By following the same path as for a point mass rotor, we finally arrive at the following equation for ball number 11 (and corresponding expressions for the other balls)

$$
\begin{aligned}
& \left(r_{0} \ddot{\varphi}_{11}+\frac{c \cdot r_{0}}{m_{b 1}} \cdot \dot{\varphi}_{11}\right) \cdot \frac{1}{\Omega^{2}}=-\sin \varphi_{11}\left(\Phi_{x} e_{0}+\Phi_{\theta} z_{1} \vartheta_{0}\right) \\
& +\left(-\sin \varphi_{11} \sum c_{1}+\cos \varphi_{11} \sum s_{1}\right) \cdot e_{0} \kappa_{1}\left(\Phi_{x}+\Phi_{\theta} \frac{m z_{1}^{2}}{J}\right) \\
& +\left(-\sin \varphi_{11} \sum c_{2}+\cos \varphi_{11} \sum s_{2}\right) \cdot e_{0} \kappa_{2}\left(\Phi_{x}+\Phi_{\theta} \frac{m z_{2}^{2}}{J}\right)
\end{aligned}
$$

\section{Balancing}

A perfect balance would require that all the quantities $e_{\xi}$, $e_{\eta}, \vartheta_{\xi}$, and $\vartheta_{\eta}$ be $=0$. The solution to this is obtained from Equation (13)

$$
\begin{aligned}
& \sum s_{1}=\sum s_{2}=0 \\
& \sum c_{1}=\frac{\kappa_{2}-\Gamma_{2}}{\kappa_{1} \Gamma_{2}-\kappa_{2} \Gamma_{1}} \\
& \sum c_{2}=\frac{\kappa_{1}-\Gamma_{1}}{\kappa_{1} \Gamma_{2}-\kappa_{2} \Gamma_{1}}
\end{aligned}
$$

which is easily transformed to values for the angles $\varphi_{11} \ldots \varphi_{21}$.

\section{Stability}

For the solution to the equilibrium Equation [17] to be valid, it must represent stable positions for the balls. By analyzing this in the same way as in the section "Perfect Balancing Ring," we finally arrive at the following conditions for stability

$$
\begin{aligned}
& \Phi_{x}+\Phi_{\theta} \frac{m z_{1}^{2}}{J}<0 \\
& \Phi_{x}+\Phi_{\theta} \frac{m z_{2}^{2}}{J}<0
\end{aligned}
$$

\section{Only One Balancing Ring}

It is obvious that a perfect balancing requires two balancing rings. If only one exists, perhaps for some practical reasons, there will be a residual unbalance. The equilibrium of the balls (balancing ring 1 is assumed to exist) will be given by equations not exactly the same as (17), but instead

$$
\begin{aligned}
& \sum s_{1}=0 \\
& \sum c_{1}=-\frac{e_{0} \Phi_{x}+\vartheta_{0} \Phi_{\theta} z_{1}}{e_{0} \Phi_{x} \kappa_{1}+\vartheta_{0} \Phi_{\theta} z_{1} \Gamma_{1}}
\end{aligned}
$$


Inserting these expressions into Equations (12) and (13) gives the motion of the rotor

$$
x=e_{0} \vartheta_{0} \Phi_{x} \Phi_{\theta} z_{1} \frac{\Gamma_{1}-\kappa_{1}}{e_{0} \Phi_{x} \kappa_{1}+\vartheta_{0} \Phi_{\theta} z_{1} \Gamma_{1}} \cos \Omega t
$$

The motion at the balancing ring is obtained by Equation (15) and gives

$$
x_{1}=0
$$

Again we note that the ring will rotate with its center stationary, and the rest of the rotor has to adjust its motion to this. This confirms the result as obtained for the eccentric ring: the only way for the balls to be at rest is when the ring is running concentrically. This means that there is no guarantee that the rotor as a whole will run smoother, even if the balancing in itself has been successful. Equation (18) may mean that the motion of the rotor center has increased.

\section{CONCLUSIONS}

By using a time-averaging technique it has been shown under what circumstances the balancing will work. Some of these result resemble what has been since long known but the results presented here are more exact. They also confirm what has been shown by direct simulations of ball motion. Some of the results are:

- For an anisotropic case with a point mass, rotor balancing will be successful above the first critical and up to the point where the amplitudes in $x$ - and $y$ directions are equal, meaning that the motion is a backward whirling circle. It is or course also stable above the second critical.

- Deviations from a perfect ring geometry will greatly aggravate the possibility to balance. In particular, an ellipticity can cause severe residual unbalance.

- The balancing will always result in the ring running with its center at rest. The necessary conditions for this to take place is when the balls are large enough to counteract the initial unbalance and the balls have a stable position.

\section{ACKNOWLEDGMENTS}

This research was made partly during a period as visiting professor at Kobe University in Japan. I am deeply indebted to professor H. Kanki and his colleagues for their collaboration and for the spiritual atmosphere I met there.

\section{NOMENCLATURE}

$\begin{array}{ll}c & \text { viscous resistance } \\ e, e_{0}, e_{\xi}, e_{\eta} & \text { unbalances } \\ i, j & \text { ball index }\end{array}$

$\begin{array}{ll}m & \text { mass of rotor } \\ m_{b} & \text { mass of ball } \\ n & \text { wave number } \\ \alpha & \text { angle for ring defect } \\ \beta & \text { angle to unbalance } \\ \gamma & \text { angle to force } \\ \theta & \text { angular motion } \\ \vartheta, \vartheta_{\xi}, \vartheta_{\eta} & \text { dynamic unbalance } \\ \kappa & r_{0} m_{b} / e_{0} m \\ \lambda & c / m_{b} \Omega=\text { nondimensional resistance } \\ \xi, \eta & \text { rotating coordinates } \\ r, r_{0} & \text { radius } \\ t & \text { time } \\ u & e_{0} / r_{0} \cdot \Phi \\ x, y, z & \text { fixed coordinates } \\ N & \text { normal force } \\ T & \text { resistance force } \\ \tau & \Omega t, \text { nondimensional time } \\ \varphi & \text { ball position } \\ \psi & \text { phase angle } \\ \omega & \text { natural frequency } \\ \Delta r & \text { ring deviation } \\ \Gamma & m_{0} z \kappa / j \vartheta_{0} \\ \Phi & \text { magnification factor } \\ \Omega & \text { rotational frequency } \\ & \end{array}$

\section{REFERENCES}

Den Hartog, J. P. 1954. Mechanical Vibrations, 4th Edition, McGraw Hill, New York.

Gosiewski, Z. 1985. Automatic balancing of flexible rotors, part I: Theoretical background. Journal of Sound and Vibration 100:551-567.

Gosiewski, Z. 1985. Automatic balancing of flexible rotors, part II: Synthesis of system. Journal of Sound and Vibration 114:103-119.

Inoue, J., Jinnouchi, Y., and Araki, Y. 1978. Dynamic behaviour of automatic alancers, Proceedings of the 8th International Conference of Nonlinear Oscillations, Prague.

Kubo, S., Jinnouchi, Y., Araki, Y., and Inoue, J. 1986. Automatic balancer (Pendulum balancer). Bulletin of the JSME 29:924-928.

Lindell, H. 1996. Vibration reduction on hand-held grinders by automatic balancing. Central European Journal of Public Health 4:4345 .

Ohtami, K., Otsuki, F., Uematsu, H., Nakamura, Y., Chida, Y., and Nishimura, O. 2001. Active mass auto-balancing system for centrifuge rotor providing artificial gravity in space, Proceedings of the 2001 ASME Design Engineering Technical Conference, Pittsburgh.

Rajalingham, C., and Rakheja, S. 1998. Whirl suppression using guided rolling balancers. Journal of Sound and Vibration 217(3):453-466.

Sharp, R. S. 1975. An analysis of a self-balancing system for rigid rotors. Journal of Mechanical Engineering Science 17(4):186-189.

Thearle, E. I. 1932. A new type of dynamic-balancing machine. Transactions of the ASME 54:131-141.

Wettergren, H. I. 2001. Auto-balance anisotropic mounted rotors, Proceedings of the 2001 ASME Design Engineering Technical Conference, Pittsburgh. 

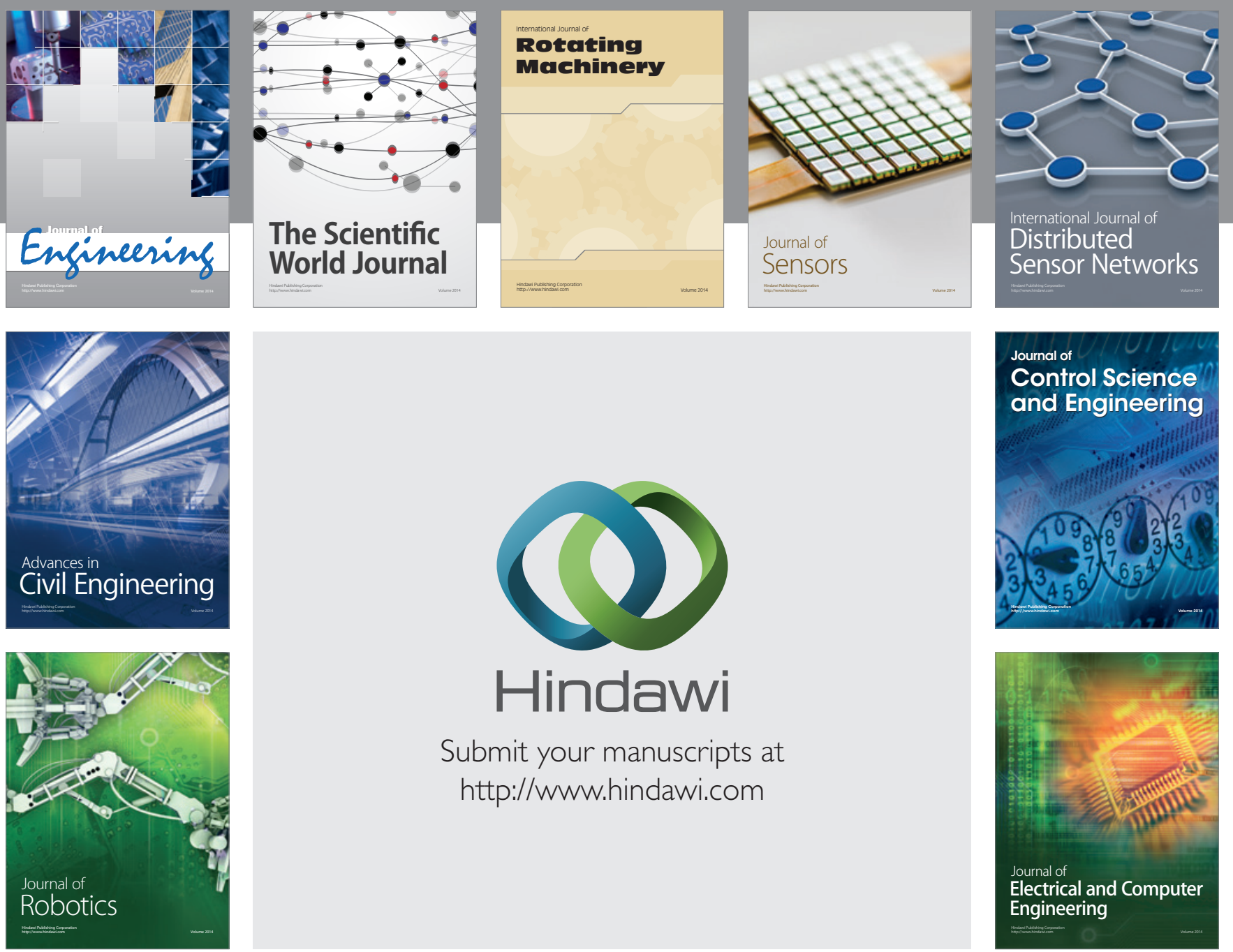

Submit your manuscripts at

http://www.hindawi.com
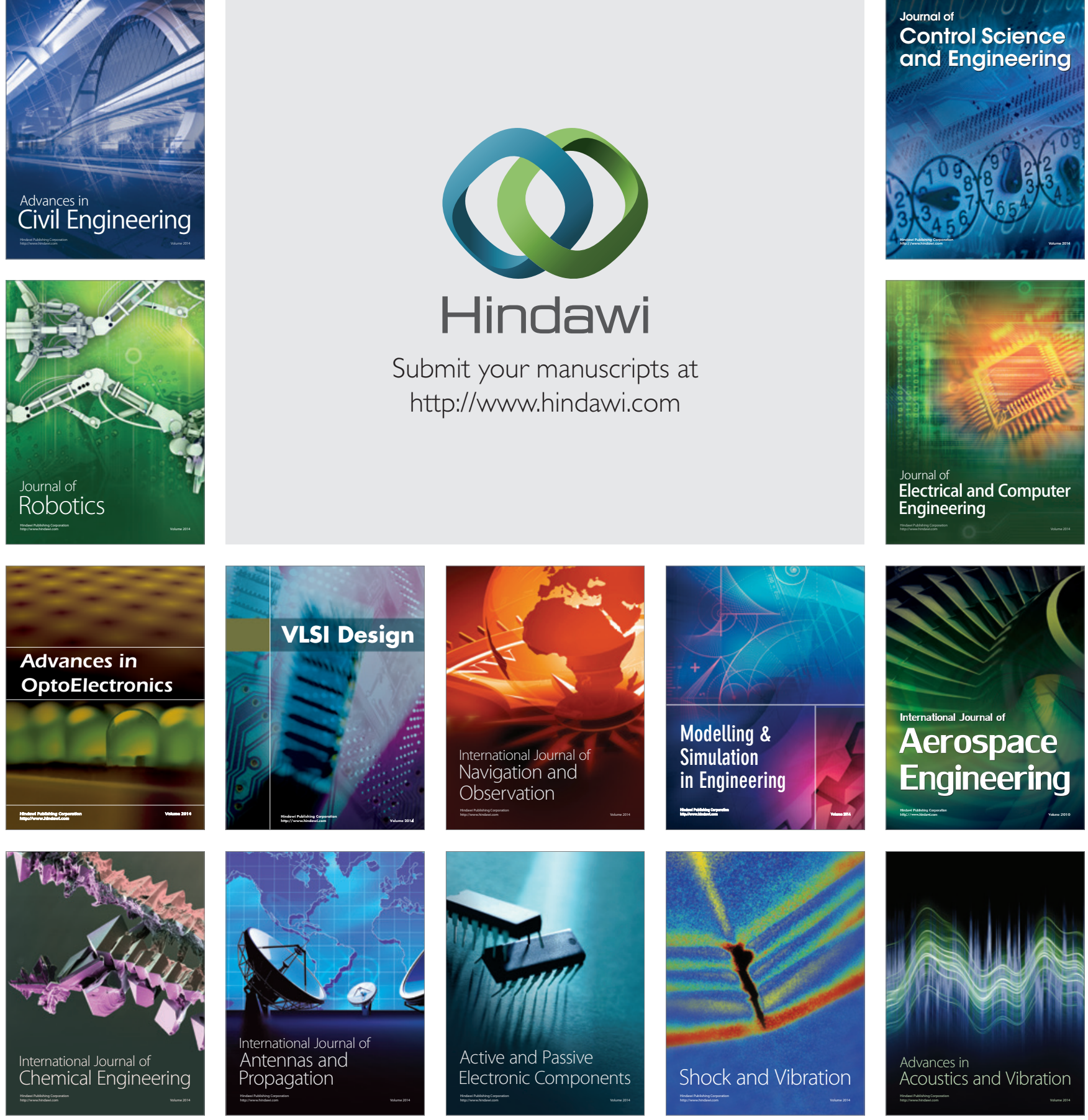